\title{
UTLLZACIÓN DEL ENTORNO DE APRENDIZAJE VIRTUAL DE LA UNIVERSIDAD COMO SOPORTE A LOS CURSOS MASIVOS EN LÍNEA Y ABIERTOS
}

\author{
Alberto Caballero Martínez ${ }^{1}$, Concepción Marcillo García 2, Jesús Soto Espinoza 1 \\ Universidad Católica San Antonio de Murcia (España) ${ }^{1}$ \\ Universidad Estatal del Sur de Manabí (Ecuador) ${ }^{2}$ \\ alberto.caballero.martinez@gmail.com
}

Cómo citar este artículo: Caballero, A., Marcillo, C. y Soto, J. (2015) Utilización del entorno de aprendizaje virtual de la universidad como soporte a los cursos masivos en línea y abiertos. CUMBRES, Revista Científica. 1 (1) 57 - 62

\section{RESUMEN}

El presente trabajo destaca los elementos más relevantes de los cursos masivos en línea y abiertos (MOOC) como recurso disponible para favorecer el desarrollo económico-social y afianzar los procesos de externalización de la Universidad en su entorno.

Los cursos de este tipo ofrecen ventajas entre las que resalta la accesibilidad al conocimiento actual generado en las Universidades. Sin embargo, la falta de contextualización de este conocimiento hace muy difícil su aplicación directa en la solución de problemas sociales y económicos de la región.

Por lo tanto, se propone un marco de trabajo para la concepción, impartición, gestión y perfeccionamiento de los cursos que desde la Universidad se podrían ofrecer en su entorno económico, social, político y cultural concreto. La idea de utilizar el Entorno Virtual de Aprendizaje de una Universidad determinada impulsa la realización de este tipo de cursos sin que ello suponga una carga adicional significativa para la Institución.

Palabras clave: Cursos masivos en línea y abiertos, entornos virtuales de aprendizaje, educación abierta, externalización de la Universidad.

\section{ABSTRACT}

This paper highlights the most relevant elements of massive open online courses (MOOC) as a very valuable resource available at universities (1) to promote economic and social development and (2) to externalize the university in its environment. MOOC offer many advantages, among others, they improve the accessibility to the most recent knowledge generated at universities. However, the knowledge offered in these courses is not contextualized into the university environment. For this reason, the application of this knowledge in order to solve real social and economic problems is not clear.

In this paper, a framework for the design, delivery, management and improvement of courses offered by universities in order to solve the nearest economic, social, political and cultural problems is proposed. Using the Virtual Learning Environment (EVA in Spanish) available at the university, the scope and the usefulness of these courses could be enhanced.

Keywords: Massive open online courses, virtual learning environments, open education, educational universalization. 


\section{INTRODUCCIÓN}

Los cursos masivos en línea y abiertos (en inglés, Massive Open Online Courses, MOOC) son una alternativa de educación abierta a la que muchos centros educativos recurren actualmente. Su idea básica es la liberación del conocimiento producido en las Universidades de manera que éste sea accesible para un público amplio y, muchas veces, fuera de su entorno.

Los MOOC se orquestan a partir de los recursos educativos abiertos (OER) que desde las Universidades son producidos-seleccionados para que cualquiera persona pueda utilizarlos. La mayoría de las veces, estos recursos están orientados para que el público descubra algo más sobre la Universidad y el conocimiento que produce, el modo en que se imparte, entre otros (Friesen, 2009).

Actualmente existen varios repositorios de OER y distintas plataformas desde las cuales se ofrecen MOOC. Entre las plataformas más populares de MOOC se encuentran Coursera, Miriada X, Udacity y Edx. Aunque la oferta es amplia y las temáticas de los cursos ofrecidos son muy variadas, de manera general, los MOOC tienen la carencia común de que se encuentran descontextualizados del entorno concreto donde se ha producido el conocimiento que se ofrece. Por lo tanto, la aplicación de dicho conocimiento no siempre es fácilmente aplicable en el contexto económico, social y cultural del estudiante que lo consume (Borrero, 2014), (Sangrá et al., 2013).

En este trabajo se proporciona un conjunto de ideas metodológicas para se ofrezcan MOOC interesantes y contextualizados en el entorno de las Universidades que los imparten. En la sección dos se analizan las fortalezas y debilidades de esta modalidad de enseñanza. Además, se relacionan los retos más significativos a los cuales se enfrentan estos cursos, a la vez que se comentan las potencialidades que podría reportar la utilización de un Entorno Virtual de Aprendizaje (EVA). Luego, en la sección tres se exponen las ideas metodológicas que constituyen la contribución principal de este trabajo, las cuales se ofrecen en un marco que define varias fases de actuación que involucra a los diferentes sectores sociales, económicos, académicos y políticos del entorno de la Universidad. La sección cuatro relaciona las ventajas, que a entender de esta propuesta, podría reportar la utilización del propio EVA de la Universidad como plataforma para los MOOC, sin la necesidad de invertir nuevos recursos y aprovechando los ya existentes. Finalmente, en la sección cinco se ofrecen las conclusiones del presente artículo.

\section{MATERIALES Y MÉTODOS}

La principal ventaja de los MOOC viene dada por su facilidad de acceso a los contenidos que trata. Por una parte, no presenta limitaciones en cuanto a la localización y movilidad geográfica de los participantes y, por lo general, tampoco grandes restricciones de tiempo. La impartición de estos cursos se desarrolla de manera asíncrona y, como mucho, solo establece límites para la realización de algunas actividades evaluativas o de liberación de nuevos contenidos.

Los MOOC constituyen la vía más sencilla de acceder a una amplia gama de contenidos actualizados, relacionados con un ámbito de conocimiento muy concreto. Es un canal que, cada vez más utilizan las Universidades para dar a conocer su oferta académica, señalando de manera muy precisa varias aplicaciones del conocimiento teórico-práctico que en ellas se generan. En algunos casos los MOOC podrían verse como un arma de doble filo por cuanto no constituyen una fuente de ingreso para las Universidades. Sin embargo, contribuyen a promocionar sus estudios reglados, relegando su actuación formal (y consecuentemente, cobrando las tasas oportunas) en la certificación de los conocimientos adquiridos.

En el marco de su labor de divulgación, los MOOC fomentan la democratización, no solo local o regional sino global, de la educación, la cual fuera mayor si la Universidad se centrara en su entorno social, económico, cultural y político más cercano. La utilidad de la enseñanza y de la investigación universitaria se hace más efectiva cuando la institución se mantiene atenta y receptiva a la demanda de los diferentes factores de su entorno. Ante dicho contexto, el presente trabajo propone un marco de referencia para potenciar la efectividad de los cursos ofrecidos que, entre otras cuestiones, considera la inclusión de varios componentes en la concepción, planificación, impartición y perfeccionamiento de los cursos.

Desde el punto de vista de los estudiantes, los MOOC constituyen una amplia fuente de recursos educativos, permitiéndoles profundizar en los temas de interés por medio de diversos enfoques ofrecidos por docentes de varias Universidades, incluso de varios países. En este sentido, el valor añadido viene dado por la organización sistémica y secuencial que los profesores ofrecen en sus cursos. En definitiva los recursos podrían existir fuera de los MOOC (incluso de las Universidades) y los estudiantes accederían a ellos de manera asíncrona, sin limitaciones de espacio.

De forma adicional, la amplia gama de OER permite a los 
estudiantes y profesores evaluar la calidad e idoneidad de los materiales, lo que promueve un proceso de perfeccionamiento continuo de los cursos y sus materiales, en aras de su efectividad en la solución a problemas concretos de los ámbitos económico y social.

La alta disponibilidad y variedad de los OER tratados en los MOOC permite a los estudiantes crear su propio plan de aprendizaje (García, 2007). Otra ventaja, y no menos importante que el resto, es la gratuidad de estos cursos, contribuyendo de manera significativa a la democratización de la educación y abriendo las puertas hacia un modelo de formación continua. No obstante, a pesar de las ventajas que puedan reportarse, este tipo de cursos presentan algunos inconvenientes provocados por su propia naturaleza.

Al ser cursos abiertos y masivos en muchas ocasiones, el contenido que tratan se encuentra descontextualizado parcial o totalmente y, por lo tanto, pueden no ser de aplicación directa en los problemas del entorno cercano a la Universidad. En la propuesta de marco de trabajo que se hace en este artículo, se parte de la premisa que involucrando a los diferentes factores del entorno universitario, se pueden conseguir cursos exentos de este problema.

Los recursos proporcionados en dichas plataformas no suelen estar adaptados a las necesidades reales de cada estudiante. Por lo general, se asume a priori que todos los alumnos tienen el mismo nivel de conocimientos previos. Sin embargo, este nivel puede ser muy dispar incluso en el manejo de las herramientas de enseñanza en línea que se utilizan.

El seguimiento en este tipo de cursos se hace muy difícil. Incidir sobre las carencias y dudas de los usuarios no es una tarea sencilla, tanto por el volumen de estudiantes como por la poca comunicación que existe entre ellos y de éstos con el profesor. Por lo general, la impartición de los MOOC se realiza de manera unidireccional, de profesor a alumno, sin ninguna retroalimentación. Una buena alternativa para favorecer las interacciones entre los participantes de un MOOC, es ofrecer herramientas de comunicación y colaboración propias de la plataforma que soporta el Entorno Virtual de Aprendizaje de la Universidad en cuestión.

De igual manera, el aprovechamiento del estudiante no puede ser constatado sin que queden sesgados otros factores ajenos a la organización del curso. Algunos de éstos están provocados por la carencia de herramientas de evaluación efectivas que permitan certificar el aprendizaje de los conocimientos.
Las dos limitantes comentadas anteriormente (baja interacción entre los participantes y la falta de retroalimentación del aprendizaje) están matizadas por la alta tasa de abandono y la constancia reducida de los estudiantes matriculados en estos cursos. Dicho problema puede mejorarse aumentando el interés del curso en el público potencial, o lo que es lo mismo, abordando cuestiones que motiven los grados de atención, valorando para ello factores sociales, económicos y políticos del entorno.

Comentadas las ventajas y desventajas, a continuación relacionamos los principales retos que, según (Sangrá et al., 2013) afectan el desarrollo de los MOOC. Para cada uno de estos retos, se plantean algunas ideas de mejora-gestión que podrían ser abordadas por una propuesta en este sentido:

Mantenimiento de un estándar de calidad ya conseguido con educación en línea, mediante la utilización de un EVA para soportar los MOOC. Podrían utilizarse procedimientos docentes ya establecidos, la gestión administrativa, los canales de comunicación, la interacción entre los participantes, entre otros.

- Diseñar y fortalecer las posibilidades de interacción entre sus participantes. En los EVA suelen existir herramientas para la mensajería privada, la realización de sesiones de chat, videoconferencias, foros de discusión, tutorías, entre otros.

- Dotar de un rol de acompañamiento potente al docente, quien debe ser el que facilite los recursos. Los EVA permiten organizar los materiales del curso, según su naturaleza, con fines académico. Su visibilidad a los estudiantes en un momento dado podría gestionarse en base a las evaluaciones superadas, participación en determinados foros de discusión, entre otros.

- Desarrollar métodos alternativos de evaluación que vayan más allá de los exámenes tradicionales en los entornos virtuales, los cuales sumen aspectos cualitativos. En este sentido, Ios EVA pueden contabilizar el nivel de utilización de la plataforma, los recursos accedidos, las herramientas utilizadas, la interacción con otros participantes, etc.

\section{RESULTADOS}

Para que los MOOC sean efectivos en cuanto a la satisfacción de las necesidades socioeconómicas presentes en el entorno de la Universidad, se propone la realización de un conjunto de actividades que involucre a todos los factores sociales, económicos, académicos y políticos. Estas actividades pueden enmarcarse en algunas de las siguientes 
fases:

- Detección de las necesidades del entorno

Identificación del conocimiento potencial

- Concepción, organización, posible reconocimiento

- Difusión del curso en el entorno

Preparación de los materiales

Impartición del curso

Evaluación de los resultados

Estas fases que se proponen podrían abordarse de manera repetitiva e incluso interactuando entre ellas.

\section{Fase 1. Detección de necesidades del entorno}

La Universidad debería dedicarse a censar periódicamente las necesidades de su entorno, actualizando así un banco de problemas accesibles para todos los profesores e investigadores. Para ello podría convocarse a los coordinadores de los organismos locales más representativos, gestores de cooperativas de producción, colegios profesionales, etc.

\section{Fase 2. Identificación del conocimiento potencial}

De la misma manera que la Universidad debe tener un banco de problemas del entorno productivo, social y cultural, tiene también que contar con un catálogo del conocimiento potencial de la propia institución. Este banco de conocimiento debería relacionar aquellos temas donde los miembros de la comunidad universitaria podrían hacer aportaciones relevantes. Por ejemplo, una manera sencilla de implementarlo sería en la elaboración de un catálogo global o directorio de expertos, donde cada profesor indique el área y tema con que contribuiría. También podrían organizarse seminarios de difusión con los principales exponentes y expertos sociales, donde se den a conocer los conocimientos necesarios a trabajar y cómo éstos contribuirían a resolver los problemas-necesidades del entorno.

\section{Fase 3. Concepción, organización y posible reconoci- miento}

El curso a proponer debe resultar del enlace de varios elementos del banco de problemas así como del repositorio de recursos de conocimientos disponibles en la Universidad. En este punto, el planteamiento del proyecto debe comenzar con reuniones de trabajo entre las personas que conocen al detalle los problemas que determinan el alcance y objetivo de un curso, junto a académicos y docentes con experticia en sus dominios de conocimiento.
Igualmente, debe valorarse el tamaño del curso. En muchos casos debería dividirse en cursos pequeños, de duración relativamente corta, favoreciendo así a la culminación de los cursos por parte de los estudiantes.

Otra cuestión interesante sería analizar la correspondencia entre los MOOC y los posibles programas reglados o certificaciones oficiales que se ofrecen en el ámbito universitario. Lo ideal sería que los MOOC estuvieran enmarcados en algún programa académico legalizado. De esta manera, un estudiante no matriculado en la Universidad podría, mediante la realización de varios MOOC, completar los créditos de un programa o certificación específica y solo recurrir a la sede institucional para examinarse y obtener la acreditación de estudios.

\section{Fase 4. Difusión del curso en el entorno}

Es imprescindible una fase de difusión tanto en el entorno económico-social como en el universitario. En dicho momento el curso debería estar disponible para que los estudiantes se matriculen y puedan acceder a las generalidades del pensum de estudio. La difusión y socialización dentro de la Universidad tiene como objetivo identificar otros expertos que puedan contribuir a este curso o a la propuesta de nuevos programas.

\section{Fase 5. Preparación de los materiales}

Por su naturaleza, los recursos utilizados en los MOOC deben ser audiovisuales y de fácil acceso. Por otro lado, antes de comenzar con la preparación de materiales debería valorarse cuáles de los recursos educativos universitarios pueden ser liberados y formar parte del repositorio de OER a disposición de las enseñanzas regladas y abiertos masivamente.

Aunque siempre se suele pensar en los materiales utilizados durante la fase de impartición del curso, también deben considerarse aquellos utilizados durante la fase de evaluación. Un ejemplo de estos recursos son los cuestionarios dedicados a la evaluación de los estudiantes a lo largo de un período lectivo.

Esta puede ser la fase de mayor trabajo del profesorado debido a que en ella se deben preparar todos los materiales y configurar el sitio del EVA dedicado a la asignatura. Sería conveniente que antes de la impartición de un programa académico, el EVA quedara configurado completamente para que el estudiante pueda valorar desde un inicio la magnitud del curso, sus objetivos, alcance, plazos, 
etc. Así, la fase siguiente dedicada a la impartición de la materia se centraría fundamentalmente en el seguimiento de los estudiantes.

\section{Fase 6. Impartición del curso}

La impartición del curso considera que previamente se ha efectuado la matrícula de los estudiantes. Bajo esta perspectiva, debería ofrecerse la posibilidad que los estudiantes puedan inscribirse por sí mismos. Además, sería conveniente que al hacerlo tengan al menos una previsualización de los contenidos y actividades más representativas, lo que favorecería la visión global de las asignaturas, sus objetivos y alcances, disminuyendo así la tasa de abandono una vez iniciado el programa.

En este proceso de matrícula y durante toda la impartición del curso sería conveniente explotar el componente participativo de la web 2.0 que se puede obtener de varios redes sociales tipo facebook y twitter. De esta manera, se estarían promoviendo las interacciones entre todos los participantes del grupo, lo que daría la posibilidad de establecer canales de comunicación informales, no reglados ni regulados por el propio EVA de la Universidad.

Concretamente en la impartición del curso, se podría aplicar la experiencia adquirida en estudios de caso sobre enseñanza en línea. Aunque todas las actividades se presuponen no presenciales, con herramientas TIC de soporte, algunas se podrían organizar síncronamente. Es decir, se citaría a los estudiantes en un momento determinado para debatir, en la medida de lo posible, algún tema concreto. Esta cuestión podría entrar en contradicción con el concepto de curso masivo. Sin embargo, se puede pensar en convocar varias reuniones con un número de participantes no muy elevado.

Aquellas actividades síncronas podrían tener como soporte las propias herramientas del EVA, entre las que destacan: videoconferencias, tutorías especializadas, chats de aclaración de dudas, etc. Por otra parte el resto de actividades asíncronas, en aras de favorecer el seguimiento y aprovechamiento del estudiante, se llevarían a cabo utilizando también las propias herramientas del EVA (foros de discusión, mensajería instantánea, cuestionarios, entre otros).

\section{Fase 7. Evaluación de los resultados}

Aunque en los $\mathrm{MOOC}$ no se emite certificado sobre la evaluación de los estudiantes, sí se pueden utilizar las herra- mientas de evaluación del EVA para:

- Brindar una valoración al estudiante sobre su desempeño.

- Ofrecer indicadores sobre el curso y los estudiantes para que el profesor pueda tomar las medidas correctoras que estime necesarias.

Por un lado, la evaluación no solo debe estar orientada al estudiante, sino también al propio curso. Y por otro lado, no debe ésta darse solo al final de un programa académico, sino que deberían plantearse varios momentos para evaluar lo aprendido.

La evaluación realizada al estudiante debería servir como retroalimentación para que quienes superen el curso puedan presentarse a los exámenes de certificación relacionados con el mismo. De ahí, que si se quiere establecer un MOOC de naturaleza atractiva debería considerarse si contribuye total o parcialmente a alguna certificación existente dentro de la Universidad. Por lo tanto, la evaluación realizada al curso tiene que servir como punto de partida para mejorar la calidad de los propios materiales de estudio así como la efectividad metodológica de las herramientas del EVA utilizadas en dicho curso.

\section{DISCUSIÓN}

La propuesta que se presenta considera además que las fases 5,6 y 7 se soportan por el propio EVA utilizado por la Universidad. De esta manera tendríamos ventajas tales como:

- Conservar los estándares de calidad, métodos de trabajo, canales de comunicación, gestión docente y administrativa, entre otros, que ya se han establecido y validado en la educación en línea

- Aprovechar las herramientas colaborativas y comunicativas del EVA

Aprovechar la capacidad del EVA para la gestión y organización de todos los materiales del curso

- Aprovechar las herramientas del EVA para recopilar y consolidar indicadores de utilización y aprovechamiento de cada uno de los componentes del propio entorno - Utilizar las herramientas de evaluación y seguimiento del estudiante ya establecidos en el EVA

- Promover el sello de identidad de la Universidad y su oferta formativa 


\section{CONCLUSIONES}

1. Los MOOC constituyen una alternativa viable y eficaz para llevar a cabo una educación abierta a la comunidad. Mediante ellos se ofrece conocimiento de alto nivel a un público amplio y, de manera general, con diferentes necesidades y demandas.

2. Es conveniente utilizar los propios entornos virtuales de aprendizaje de las Universidades para ofrecer dichos cursos. De esta manera, se aprovechan los recursos ya disponibles y generados, sin que dichos cursos supongan una sobrecarga adicional para las instituciones de enseñanza.

\section{REFERENCIAS BIBLIOGRÁFICAS}

- Borrero, J. (2014). Mientras los académicos muestran escepticismo frente a los "MOOC", Las Universidades continúan apostando por ellos. Revista Educación Virtual, http://revistaeducacionvirtual.com/archives/783.

- Friesen, N. (2009). Open Educational Resources: New Possibilities for Change and Sustainability. International Review of Research in Open and Distance Learning. Vol. 10 (5)

- García, L. (2007). El aprendizaje fuera de la escuela. Boletín Electrónico de Noticias de Educación a Distancia (BENED), http://e-spacio.uned.es/fez/eserv/bibliuned:20107/aprendizajefuera.pdf.

- Sangrá, A. \& Wheeler, S. (2013). Nuevas formas de aprendizaje informales: ¿O estamos formalizando lo informal? En: La informalización de la educación. Revista de Universidad y Sociedad del Conocimiento (RUSC). Vol. 10 (1), p. (107-115), http://dx.doi.org/10.7238/rusc. v10i1.1689. 